\title{
Angioplastia a puente venoso por vía radial izquierda distal. Nueva alternativa de acceso arterial
}

\author{
Saphenous vein graft angioplasty by left distal radial access. A new arterial \\ access alternative
}

Leandro Lasave' ${ }^{1}$, Maximiliano Rossi', Fernando Kozak' , Aníbal Damonte' ${ }^{1}$ Eduardo Picabea'

\section{RESUMEN}

La vía radial se ha convertido en el acceso de elección para los procedimientos coronarios. Si bien el acceso derecho es el más utilizado, en ciertas situaciones el acceso izquierdo es necesario. En estos casos, la vía de acceso distal, por punción de la arteria radial a nivel de la fosa radial, tiene ciertas ventajas y mayor comodidad con respecto a la vía de acceso proximal. Presentamos el caso de un paciente con síndrome coronario agudo y cirugía de revascularización miocárdica previa, sometido a angioplastia de puente venoso a arteria circunfleja a través de acceso radial izquierdo distal.

Palabras claves: arteria radial, intervención coronaria percutánea, stents.

\begin{abstract}
Radial access has become the first choice approach in coronary procedures. Although the right radial access is the most commonly used, there are some situations in which left approach is necessary. In these cases, the radial approach via the left distal radial access at the anatomical snuffbox is more comfortable than conventional radial access and it brings advantages to the patients and operators. We present a case of a patient with acute coronary syndrome and previous CABG underwent coronary angioplasty of safenous vein graft to circunflex by left distal radial access.
\end{abstract}

Key words: radial artery, percutaneous coronary intervention, stents.

Revista Argentina de Cardioangiología Intervencionista 2018;9(1):56-59. Doi: 10.30567/RACI/201801/0056-0059

\section{INTRODUCCIÓN}

Desde hace varios años la vía radial es considerada el acceso de elección para los procedimientos corona$\operatorname{rios}^{1-3}$. Además de ser más confortable para el paciente ya que permite una rápida movilización y deambulación, la mayor ventaja sobre la vía femoral se basa en términos de seguridad, con menor tasa de sangrado, incluso con menor mortalidad en situaciones especiales como en los síndromes coronarios agudos (SCA $)^{4-6}$. $\mathrm{El}$ acceso radial convencional se realiza a través del segmento proximal de la arteria derecha manteniendo la palma de la mano en supinación, dos centímetros por encima de la apófisis estiloides del radio, fundamentalmente por la posición de trabajo más confortable para el operador que esto determina. Sin embargo, cuando la vía radial izquierda debe ser utilizada, el acceso convencional genera incomodidad para el operador, tanto en la punción como durante la manipulación de los catéteres.

\footnotetext{
1. Departamento de Hemodinamia e Intervenciones Cardiovasculares del Instituto Cardiovascular de Rosario.

$\triangle$ Correspondencia: Dr Leandro Lasave. Bv Oroño 450, S2000DSR Rosario, Santa Fe, Rep. Argentina.lasavel@icronline.com
}

La utilización de la vía radial izquierda distal (VRID) a través del acceso a nivel de la fosa radial (tabaquera anatómica) permite mejorar la posición del operador y del paciente y disminuir la exposición a la radiación.

\section{SITUACIÓN CLÍNICA}

Paciente varón, 85 años, hipertenso y dislipémico. Con antecedente de cirugía de revascularización miocárdica (CRM) en 1998 con puente de mamaria interna izquierda (MII) a descendente anterior (DA) y puente venoso a circunfleja (PV-CX). Portador de angina crónica estable CF II, evoluciona con progresión de la sintomatología y consulta por episodio de angina en reposo. Es hospitalizado con diagnóstico de SCA, con gradiente de troponina T ultrasensible (32,2-182,6 unidades). En el ECG se observa infradesnivel del segmento ST de V4-V6. Luego de iniciar tratamiento antiisquémico y antitrombótico es sometido a coronariografía (CCG). Para minimizar el riesgo en la cateterización del puente MII-DA se opta por el acceso radial izquierdo, y se escoge la VRID para mayor confort del paciente y del operador.

\section{PROCEDIMIENTO}

Se realiza la asepsia-antisepsia habitual sobre la zona a punzar. Se coloca la mano izquierda del paciente so- 


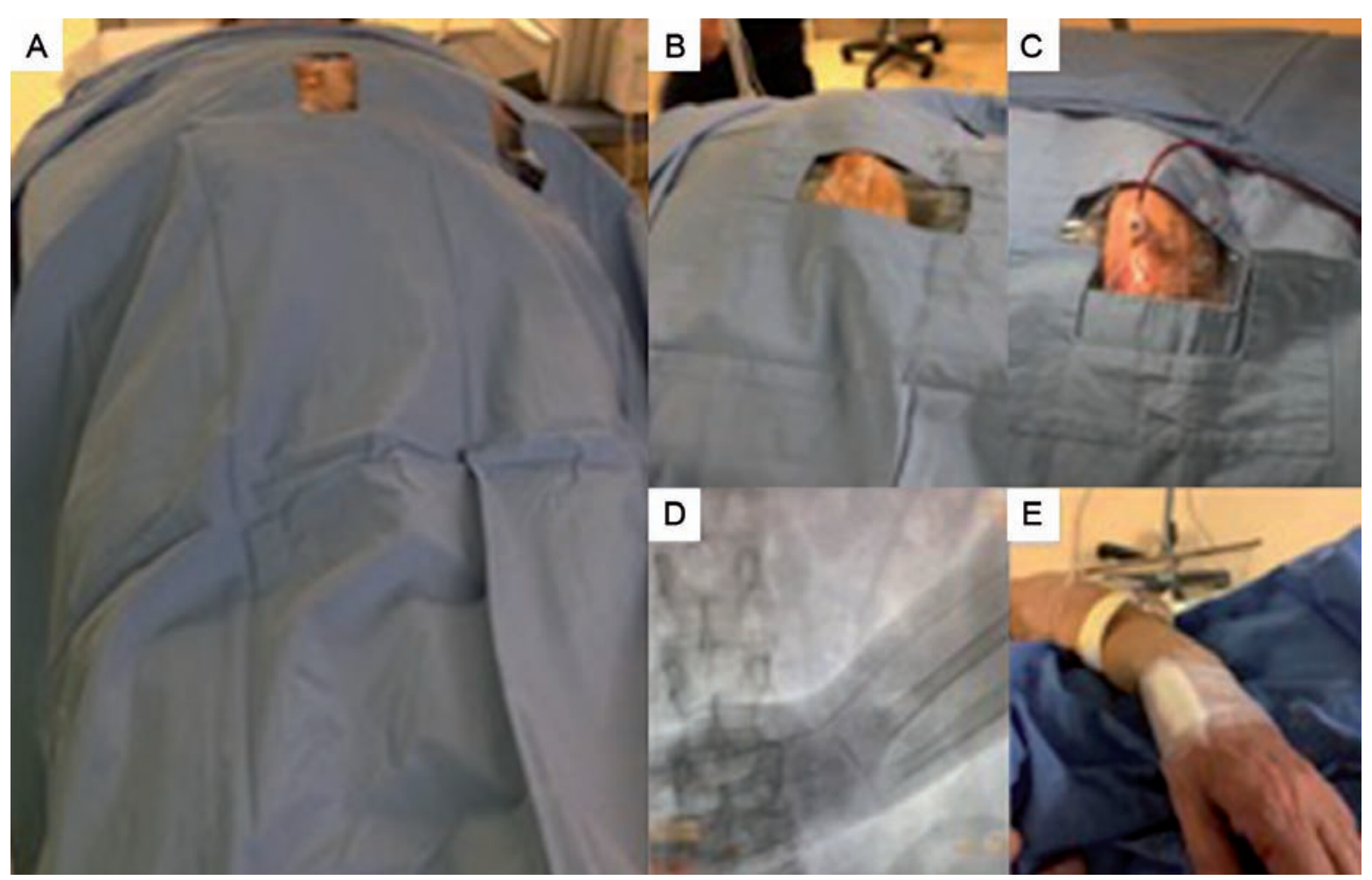

Figura 1. Preparación del campo quirúrgico. Posición de la mano izquierda del paciente a nivel de su ingle derecha (A-B). Implante de introductor $5 F(C)$. Avance de la guía e introductor sobre la arteria radial, bajo fluoroscopia (D). Vendaje semicompresivo luego de retirado el introductor (E).

bre su ingle derecha, con la palma hacia abajo (Figura 1 A-B). Se inyecta sobre la zona 3 cc de xilocaína; luego se realiza una jareta con bisturí en la parte proximal de la fosa radial, para facilitar el acceso del introductor y se punza con una aguja G20 (Terumo) la arteria radial distal, luego del origen de la rama palmar superficial (RPS) y antes de la emergencia de la rama principal del pulgar (RPP). Una vez conseguido flujo pulsátil, se posiciona una cuerda 0,025 " a nivel de la arteria radial (Figura 1D), sobre esta cuerda se avanza un introductor 5F (Terumo) (Figura 1C) y se inyectan $0,2 \mathrm{mg}$ de nitroglicerina y 2500 UI de heparina. Para el procedimiento diagnóstico se utilizó un catéter Judkins Left 4.0 5F $\left(\right.$ MeritMedical ${ }^{\mathrm{m}}$ ) para la arteria coronaria izquierda y un catéter Judkins Right (JR) 4.0 5F (MeritMedical ${ }^{\text {Dx}}$ ) para la arteria coronaria derecha, el PV-CX y el MIIDA. La CCG evidenció lesión severa en el cuerpo del PV-CX y lesión suboclusiva en la anastomosis distal del mismo (Figura 2) con flujo enlentecido. Tratándose de un SCA de alto riesgo. se decidió revascularización ad hoc. Se utilizó un catéter terapéutico JR 4.05F (Launcher, Medtronic) y se adicionaron 6500 UI de heparina. Se atravesó la lesión con una cuerda 0,014" BMW (Abbott). Luego de preparar la lesión distal con balón Maverick $^{\mathrm{m} 2}$ (Boston SC) $2,5 \times 20 \mathrm{~mm}$ se implantó un stent con liberación de zotarolimus (Resolute-Integrity, Medtronic) de 3,5×30 mm (Figura 3A), posdilatado con balón Quantum ${ }^{\text {mix }}$ Maverick $^{\text {mox }}$ (Boston SC) de $4,0 \times 15 \mathrm{~mm}$. A continuación se abordó la lesión del cuerpo del PV-CX mediante el implante en forma directa de un stent con liberación de zotarolimus (Reso-
lute-Integrity, Medtronic) de 4,0×12 mm (Figura 3B), posdilatado con balón Quantum ${ }^{\text {Tw }}$ Maverick $^{\text {mo }}$ (Boston SC) $4,5 \times 8 \mathrm{~mm}$. Las imágenes obtenidas con el software StentBoost (Philips) demuestran aceptable expansión de ambos stents. El flujo final se encuentra conservado y no se observa lesión residual (Figura 3 C-D).

Una vez finalizado el procedimiento, se retira el introductor y se realiza un vendaje manual, que no involucra la articulación de la muñeca y permite su movilidad (Figura 1E).

\section{DISCUSIÓN}

Con este caso presentamos una nueva forma de acceso arterial factible y seguro para procedimientos coronarios o vasculares, a través del segmento distal de la arteria radial izquierda.

Habitualmente la vía radial derecha, en el segmento proximal de la arteria y antes del origen de la RPS, es el acceso de elección. Sin embargo, en muchos casos la vía izquierda debe ser utilizada: radial derecha ocluida o utilizada para CRM, arteria subclavia lusoria, necesidad de canular fácilmente la arteria MII, radial derecha con tortuosidad, falla o utilización previa del acceso derecho y preferencia del paciente, sobre todo en diestros.

La vía radial izquierda es tan segura y eficaz como la derecha ${ }^{7}$, aunque es menos confortable para el operador, sobre todo en pacientes obesos donde el operador debe inclinarse sobre el paciente para maniobrar los catéteres lo que determina mayor incomodidad y mayor exposición a la radiación. 


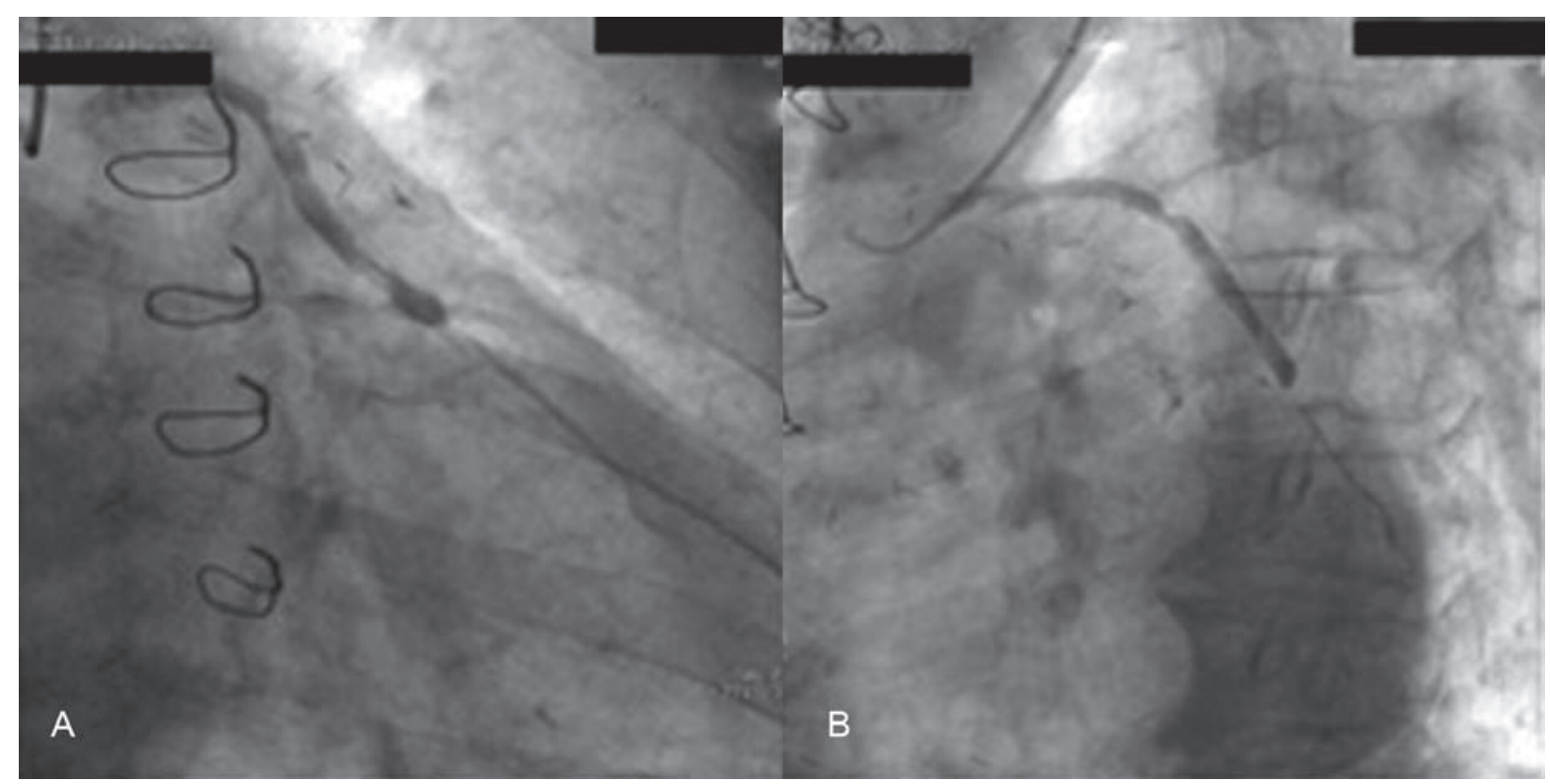

Figura 2. Coronariografía selectiva de PV-CX. Proyección oblicua anterior derecha caudal (A) y oblicua anterior izquierda (B). Se observa lesión severa focal en tercio medio del puente venoso y suboclusiva en anastomosis distal.

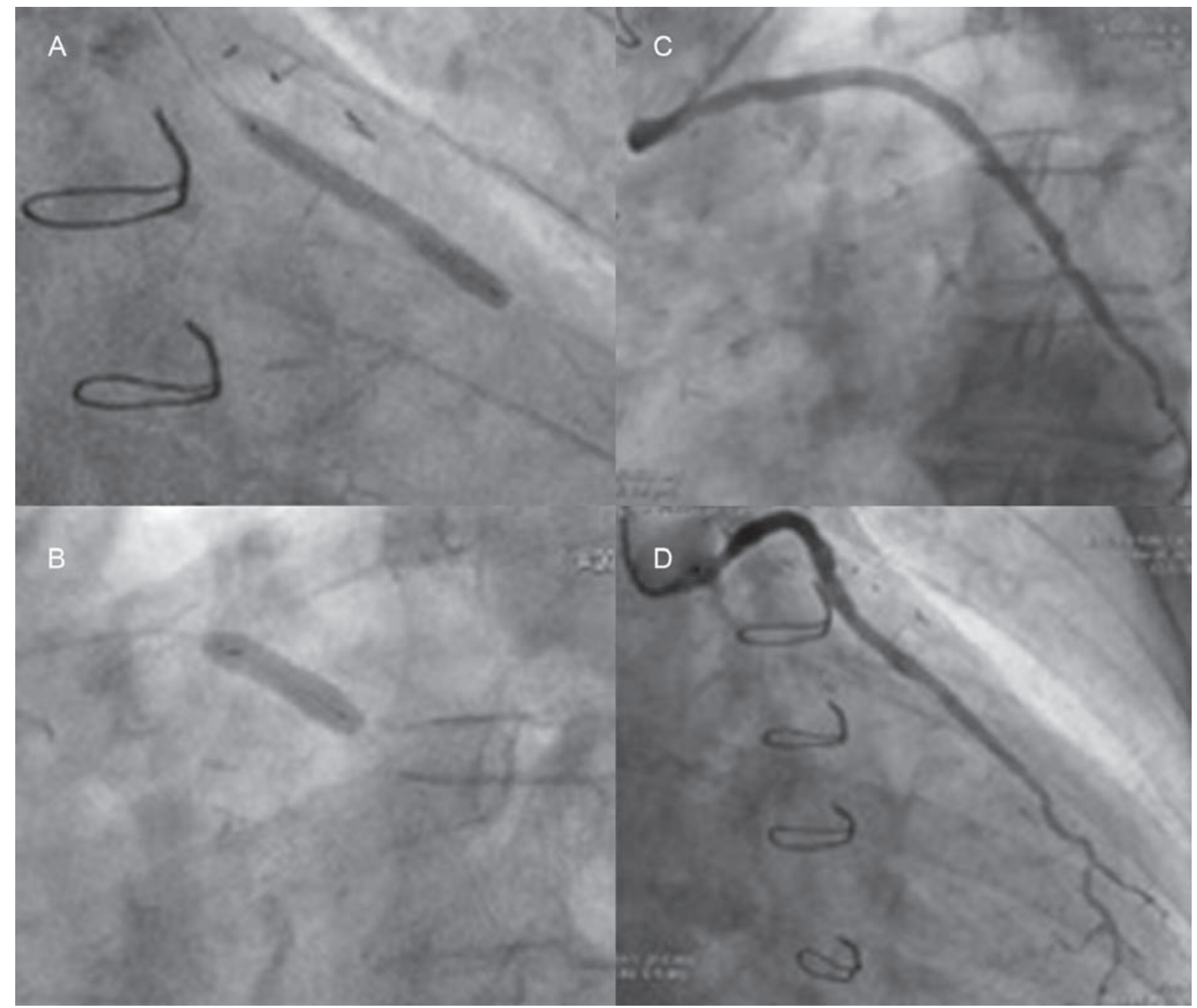

Figura 3. Angioplastia coronaria. Implante de stent en lesión distal (A) y en tercio medio del PV-CX (B). Coronariografia final: Proyección oblicua anterior izquierda (C) y oblicua anterior derecha (D). 
La utilización de la VRID, a través del acceso a nivel de la fosa radial, permite mayor comodidad del operador y del paciente y menor exposición a la radiación durante todo el procedimiento. El paciente no necesita mantener la mano en supinación ya que esta se apoya sobre su ingle y el operador adopta la posición de trabajo habitual del acceso femoral. Asimismo, el acceso izquierdo permite la utilización de los catéteres designados para el acceso femoral, ya que la entrada a la aorta ascendente es similar.

La fosa radial es el espacio hueco que se forma cuando el dedo pulgar está en extensión, delimitado hacia atrás por el tendón del extensor largo del pulgar y hacia adelante por los tendones del extensor corto del pulgar y del abductor largo. La arteria radial cruza el piso de esta fosa (tabaquera anatómica) que está formado por los huesos escafoides y trapecio ${ }^{8}$. La punción de la arteria se realiza luego de la emergencia de la RPS y antes de la RPP, lo que determina 2 importantes ventajas con respecto a la vía proximal:

- Posibilidad de su uso en situaciones de espasmo severo de la arteria radial proximal. La presencia de la RPS, a través del arco palmar, permite que el flujo de la arteria en la fosa radial se encuentre conservado y pulsátil, incluso cuando existe ausencia de pulso proximal luego de intentos fallidos de punción proximal.

- Al realizarse la punción luego del origen de la RPS se evita la posible oclusión trombótica retrógrada de la arteria radial ya que utiliza esta rama como vía de salida y a través del arco palmar superficial se mantiene permeable la RPP y la rama radial del índice?.

\section{BIBLIOGRAFÍA}

1. Kiemeneij F, Laarman GJ, Odekerken D, et al. A randomized comparison of percutaneous transluminal coronary angioplasty by the radial, brachial and femoral approaches: the access study. I Am Coll Cardiol. 1997;29:1269-75.

2. AgostoniP,Biondi-Zoccai GG, de Benedictis ML, et al. Radialversusfemoral approach forpercutaneous coronary diagnosticandinterventional procedures; Systematic overview and meta-analysis of randomized trials. J Am Coll Cardiol. 2004;44:349-56.

3. Hamon M, Pristipino C, Di Mario C, et al; Europea. Consensus document on the radial approach in percutaneous cardiovascular interventions: position paper by the European Association of Percutaneous Cardiovascular Interventions and Working Groups on Acute Cardiac Care and Thrombosis of the European Society of Cardiology. Eurolntervention. 2013;8:1242-51.

4. Valgimigli M, Gagnor A, Calabró P, et al, for the MATRIX investigators. Radial versus femoral access in patients with acute coronary syndromes undergoing invasive management: a randomised multicentre trial. Lancet 2015; 385:2465-76.
La limitación más importante de la vía distal es que la arteria es de menor calibre, por lo que la punción es más dificultosa y requiere una curva de aprendizaje. En una serie publicada, sobre 118 casos, 23\% no fueron candidatos a esta vía y de los pacientes seleccionados, en el $11 \%$ no se consiguió acceder por problemas de punción o progresión de la guía, mientras que en el resto se consiguió exitosamente el acceso distal sin complicaciones?.

Algunos operadores, para mayor seguridad, realizan la punción guiada por ecografía. En la experiencia de nuestro centro únicamente utilizamos la guía fluoroscópica. Aunque en nuestro caso se utilizó material de $5 \mathrm{~F}$, es posible el uso de sistemas de 6 y $7 \mathrm{~F}^{9}$.

Si bien no existen grandes estudios, las complicaciones reportadas son escasas y se limitan a hematomas del sitio de punción. La tasa de oclusión de la arteria en el segmento distal es menor al $2 \%{ }^{10}$. Igualmente, en caso de oclusión de este segmento, la posibilidad de isquemia del dedo pulgar o índice es baja ya que se mantiene la permeabilidad del arco palmar superficial'.

\section{CONCLUSIÓN}

La VRID es una alternativa segura de la vía convencional, sobre todo en situaciones en que el acceso izquierdo es el indicado. Existen algunas ventajas para el paciente y el operador aunque no hay estudios aleatorizados. Es recomendable tener presente esta vía como posibilidad de acceso para procedimientos diagnósticos o terapéuticos.
5. MehtaSR, JollySS, Cairns J, etal, forthe RIVAL investigators. Effects of Radia Versus Femoral Artery Access in Patients With Acute Coronary Syndromes With or Without ST-Segment Elevation. J Am Coll Cardiol. 2012;60:2490-9.

6. Bernat I, HorakD, Stasek J, et al. ST-Segment Elevation Myocardial Infarction Treated by Radial or Femoral Approach in a Multicenter Randomized Clinical Trial. The STEMI-RADIAL Trial. J Am Coll Cardiol. 2014;63:964-72.

7. Shah RM, Patel D, AbbateA, et al. Comparison of transradial coronary procedures via right radial versus left radial artery approach: A meta-analysis. Catheter Cardiovasc Interv. 2016;88:1027-33.

8. Cerda A, del Sol M. Anatomical Snuffbox and it Clinical Significance: A Literature Review. Int. J. Morphol. 2015; 33:1355-60.

9. Kiemeneij F. Left distal transradial access in the anatomical snuffbox for coronary angiography (IdTRA) and interventions (IdTRI). Eurolntervention 2017;13:851-857

10. Kaledin A. New access to facilitate endovascular operations: first-in-man study[Abstract]. Abstract of EuroPCR 2014. Euro 14A-OP033 